\title{
Autophagy dark genes: Can we find them with machine learning?
}

Tudor I. Oprea, , $, 2,3,4,5$ Jeremy J. Yang, ${ }^{1}$ Daniel R. Byrd, ${ }^{1}$ and Vojo Deretic ${ }^{3}$

1. Department of Internal Medicine, University of New Mexico School of Medicine, Albuquerque, NM, USA.

2. UNM Comprehensive Cancer Center, Albuquerque, NM, USA.

3. Autophagy Inflammation and Metabolism Center of Biomedical Research Excellence, University of New Mexico Health Sciences Center, Albuquerque, NM, USA

4. Department of Rheumatology and Inflammation Research, Institute of Medicine, Sahlgrenska Academy at University of Gothenburg, Gothenburg, Sweden.

5. Novo Nordisk Foundation Center for Protein Research, Faculty of Health and Medical Sciences, University of Copenhagen, Copenhagen, Denmark.

\section{Abstract}

Identifying novel genes associated with autophagy (ATG) in man remains an important task for gaining complete understanding on this fundamental physiological process. A machine-learning guided approach can highlight potentially "missing pieces" linking core autophagy genes with understudied, "dark" genes that can help us gain deeper insight into these processes. In this study, we used a set of 103 (out of 288 genes from the Autophagy Database, ATGdb), based on the presence of ATG-associated terms annotated from 3 secondary sources: GO (gene ontology), KEGG pathway and UniProt keywords, respectively. We regarded these as additional confirmation for their importance in ATG. As negative labels, we used the OMIM list of genes associated with monogenic diseases (after excluding the 288 ATG-associated genes). Data associated with these genes from 17 different public sources were compiled and used to derive a Meta Path/XGBoost (MPxgb) machine learning model trained to distinguish ATG and non-ATG genes (10-fold cross-validated, 100-times randomized models, median $A \cup C=0.994+/-0.0084$ ). Sixteen ATG-relevant variables explain $64 \%$ of the total model gain, and $23 \%$ of the top 251 predicted genes are annotated in ATGdb. Another 15 genes have potential ATG associations, whereas 193 do not. We suggest that some of these 193 genes may represent "autophagy dark genes", and argue that machine learning can be used to guide autophagy research in order to gain a more complete functional and pathway annotation of this complex process. 


\section{Introduction}

Autophagy (ATG) is a cytoplasmic homeostatic process defined by a suite of ATG genes conserved from yeast to man [1]. Autophagy keeps a cellular complement of organelles in a functional state and removes protein aggregates, invading pathogens, and other cargo, by capturing them and delivering them to lysosomes for degradation [1]. Autophagy is consequential for a wide array of physiological and pathological states including neurodegenerative and other degenerative disorders, cancer, chronic inflammatory illnesses and infectious diseases [2]. Mechanistically, the process of autophagy is governed by ATG genes, encoding the core autophagy machinery with most components conserved from fungi to humans [1]. However, recent studies have increasingly emphasized the existence of systems controlling and executing autophagy in mammalian cells that are quite different from those in yeast [3]. Among wellaccepted examples, are several autophagy factors absent in yeast that have been identified in organisms from C. elegans to H. sapiens, including ATG101 [4], FIP200 [5], VMP1 [6], EPG5 [7], Stx17 [8,9], and TMEM41B [10-12].

Completing the autophagy puzzle requires the systematic identification of all genes associated with autophagy. This on-going endeavor is boosted in part by the growing interest in the role of autophagy in ageing regulation [13,14] and lifespan extension [15]. ATG genes have been used in supervised machine learning models applied to ageing research [16] and are among the top features in models for predicting lifespan-extending chemicals [17]. Numerous studies have used machine learning (ML) methods to infer gene-disease associations [18-21]. However, the use of ML models to guide further autophagy research has not been previously discussed. Here we report the development of a specific ML model to predict "autophagy dark genes", i.e., understudied proteins [22] that may play a significant role in autophagy.

\section{Materials and Methods}

\section{Building Knowledge Graphs}

Seventeen different data sources, totaling over 262.3 million data points, summarized in Table 1, are used to build Knowledge Graphs (KGs). These 17 protein- and gene- centric data sources are integrated into formal data representation systems based on KGs, with typed nodes and edges, which enable the use of network-based analytical algorithms. Whereas most of the data in Table 1 refer to human proteins, we use gene orthology relationships from eggNOG [23] and InParanoid [24] to fuse rat (RGD) and mouse (IMPC, International Mouse Phenotype Consortium) model organism data into a single "pseudoprotein" that enables network-based inferences for function and phenotype across organisms. The KGs are implemented as a PostgreSQL db for performance, convenience, and integration with existing tools. Metapaths are rigorously defined via schema and templated SQL. The initial prototype was primarily coded in R, which was migrated to Python for enhanced functionality and integration options, e.g., visualization tools. The code and documentation are publicly accessible at https://github.com/unmtransinfo/ProteinGraphML (new Python package) and 
https://github.com/unmtransinfo/metap (R code). A snapshot of the currently used dataset for building KGs is available at http://pasilla.health.unm.edu/x/metap-pgdump.sql.gz.

Table 1. Data resources used to inform the development of ML-ready knowledge graphs

Data Source

CCLE[25]

GTEx[26]

HPA[27]

Reactome[28]

KEGG[29]

STRING[30]

$\mathrm{GO}[31]$

InterPro[32]

ClinVar[33]

GWAS Catalog[34]

OMIM[35]

UniProt[36] Disease

DISEASES[37]

NCBI Homology [38]

IMPC[39]

RGD[40]

LINCS[41]
Data Type

Gene expression

Gene expression

Gene \& Protein expression

Biological pathways

Biological pathways

Protein-Protein interactions

Biological pathways \& Gene function

Protein structure and function

Human Gene - Disease/Phenotype associations

Gene - Disease/Phenotype associations

Human Gene - Disease/Phenotype associations

Human Gene - Disease/Phenotype associations

Gene - Disease associations from text mining

Homology mapping of human/mouse/rat genes

Mouse Gene - Phenotype associations

Rat Gene - Phenotype associations

Drug induced gene signatures

\section{Data Points}

$19,006,134$

$2,612,227$

949,199

303,681

27,683

$5,080,023$

434,317

467,163

881,357

54,360

25,557

5,365

44,829

70,922

$2,153,999$

117,606

$230,111,315$ 


\section{Meta Path/XGBoost using Knowledge Graphs}

The majority of biological system networks (BSN) are heterogeneous with multiple node and edge types, as illustrated by the Data Types in Table 1. Recent developments in heterogeneous[42,43] and BSN [44] relationship predictions introduced and formalized a new framework that takes into account BSN heterogeneity by defining type specific node-edge paths or Meta Paths[45]. A meta path $[42,46]$ is a path consisting of a sequence of relations defined between different object types (i.e., structural paths at the meta level). In biology, the Meta Path approach can be used to seek different network paths that connect proteins / genes to specific properties such as phenotype or disease [45]. In this paper, we distinguish Meta Path (the method) from "meta paths", used to evaluate network topology. The objects in question are graph Nodes, which can be genes or proteins; diseases or phenotypes; chemical structures or drugs; and other entities relevant to modeling biomedical processes. "Structural paths" reflect relationships between the different entities, i.e., Nodes. "Paths", or Edges in topological terms, can be expression data; pathways; LINCS genomic signatures; protein-protein interactions (PPIs); or other relationships of biological relevance. Enumeration of all network paths along a defined meta path are used to compute graph topological features such as path counts by counting all path instances along that meta path. Degree weighted path counts (DWPCs - see Eq. 1) [44] use the number of Edges connecting each Node along a meta path to assign different weights to each path instance. Specifically, DWPCs quantify meta path prevalence using a dampening exponent ( $w$, set to 0.4 ) to down weight paths through high-degree nodes when computing DWPCs:

$$
D W P C=\sum_{\text {path } \in \text { Paths }}\left(\prod_{d \in D_{\text {path }}} d^{-W}\right)
$$

where $\Pi$ is the path-degree product calculated by: 1) extracting all edge-specific degrees along the path $D_{\text {path }}$, where each edge contributes two degrees; 2 ) raising each degree $d$ to the $-w$ power, where $w$ is the dampening exponent; 3 ) multiplying all exponentiated degrees to yield $П$. DWPC is the sum of path degree products $\Pi$ (the DWPC section was adapted from [44]). The Meta Path formalism enables the transformation of the heterogeneous KG data types into an ML-ready input. The algorithmic workflow of applying ML to the Meta Path methodology is depicted in Fig. 1.

\section{Insert Figure 1}

Figure 1. Analytical algorithms workflow for ranking target gene lists.

To better understand and predict "function" or "role in disease/phenotype", we selected XGBoost [47], an award-winning $\mathrm{ML}$ algorithm that can be directly deployed onto typed meta paths. XGBoost classifiers have certain advantages compared to other ML algorithms: i) very fast model training and prediction, and high scalability to very large datasets; ii) variable selection is driven by model performance as opposed to random, such as that used in comparable $\mathrm{ML}$ algorithms; iii) built-in model interpretability. XGBoost outputs variable importance in projection (VIP) estimates, unlike neural networks and other deep learning methods. Using VIP, meta-paths are sorted in decreasing order of importance, which leads to directly 
interpretable insights into mechanistic processes and Node interactions that contribute to protein/gene "function" or "role in disease." The combination of Meta Path/XGBoost (herein referred to as "MPxgb") processes assertions/evidence chains of heterogeneous biological data types and identifies similar assertions. These determine the strength of the evidence linking a gene/protein to a disease or phenotype or, in this instance, autophagy.

\section{Development of an ATG-specific MPxgb Model}

We extracted data from 17 protein-centric datasets available collected the TargetCentral relational database, TCRD [48], and summarized in Table 1. Specifically for this study, we started with 288 human genes from a specialized resource, the Autophagy database, ATGdb [49], which were processed and sorted for uniqueness. These genes were further queried for further confirmation of their ATG association using the "hsa04140" keyword in the Kyoto Encyclopedia of Genes and Genomes, KEGG[29] Pathway; using the gene ontology (GO) [31] term "GO:0061919"; or the UniProt [36] keyword "autophagy", respectively. The secondary query, used to confirm the importance of specific genes in autophagy, resulted in $\mathrm{N}=103$ genes (Supplementary Material).

We trained the MPxgb model using these 103 genes as "positive" labels, i.e., known to be associated with ATG. A separate set of $\mathrm{N}=3,468$ OMIM genes, which are associated with a variety of monogenic diseases and are not present in the ATGdb, were assumed to lack ATG involvement. We generated 100 randomized MPxgb models, using 10-fold cross-validation; the median area under the curve (AUC) for these models was $0.994 \pm 0.008$. The model with the highest AUC $(0.999932263)$ was selected to predict novel ATG genes, and is discussed here. The Excel file with the complete training set $(3,571$ genes, both positive and negative labels) is available as Supplementary Material.

\section{Results and Discussion}

This paper introduces an autophagy-dedicated machine learning model, which is intended to serve as guidance for extending the current autophagy knowledge to gene sets that may have not been previously explored. Its main purpose is to combine current levels of evidence, and show how such evidence can be captured and modeled using a binary classifier based on the Meta Path (Figure 2) and XGBoost approach, built upon 17 different data sources (Table 1). Biologically, this model implicates a set of up to 193 previously untested genes, some of that may have a functional role in autophagy.

For this model, data for 103 ATG-associated genes were contrasted with similar data for 3,468 "negative" genes, extracted from seven distinct categories of evidence: gene \& protein expression (CCLE, the cancer cell line encyclopedia [25]; GTEx, Gene Tissue Expression [26]; and the Human Protein Atlas [27]); biological pathways and gene function (Reactome [28], KEGG [29] and GO [31]); Protein-Protein Interactions (STRING [30]); protein structure and function (InterPro [32]); human gene -

disease/phenotype associations (ClinVar [33], GWAS Catalog [34], OMIM [35], UniProt Disease [36], and DISEASES [37]); animal gene - phenotype associations (mouse data from IMPC [39] and rat data from 
RGD [40], mapped to human genes via the NCBI Homology [38] resource); and drug-induced gene signatures from the Library of Integrated Network-Based Cellular Signatures, LINCS [41], respectively.

From these resources, meta paths were assembled, such as: $\{$ Protein - (member of $) \rightarrow$ STRING PPI Network $\leftarrow$ (member of) — Protein — (associated with) $\rightarrow$ Disease $\}$ and \{Protein — (expressed in) $\rightarrow$ Tissue/Cell line $\leftarrow$ (associate with) — GO Term\}. The first BSN application [44] to model novel disease associations using meta paths used logistic regression and ridge logistic regression. We adapted the Meta Path framework to XGBoost, a tree-boosting classification algorithm [47] used in 17 of the 29 winning solutions in Kaggle challenge competitions [50]. Combining XGBoost, a scalable ML algorithm capable of handling sparse data with BSN typed meta-paths; we used the MPxgb approach to predict novel protein functions given a variety of (unrelated) data sources.

The methodology introduced in this work has substantial advantages over simply applying $M L$ to an existing, static dataset. KG construction is informed by expertise spanning several biomedical domains, with automation tools designed for an online learning paradigm to incorporate the latest findings. Predictions presented here are not generated ex nihilo but from the rigorously aggregated findings of autophagy research to date. Our methodology uses powerful $\mathrm{ML}$ tools to abstract that collective intelligence and is subject to change, and improvement as the field continues to advance.

\section{Model Validation through Variable Importance Selection}

The top 40 features (total gain, 0.9) used to derive the best MPxgb ATG model are depicted in Figure 2, and available as Supplementary Material. There are 11 protein-protein interactions (PPIs), with a cumulative gain of 0.445; 4 interpro domain, IPR terms and one UniProt term (cumulative gain, 0.195); 9 GO terms (cumulative gain, 0.182); 9 LINCS terms (cumulative gain, 0.044 ); 3 pathway terms (cumulative gain, 0.017); and 3 expression terms (cumulative gain, 0.016), respectively. Using PPIs and structural terms provides a $64 \%$ contribution to the ATG MPxgb model; by contrast, pathway and expression terms. The presence of "autophagy" in $6 \mathrm{GO}$ terms and one UniProt term provides a cumulative gain of 0.154 . The related terms, "autophagy of mitochondrion"; "autophagy of nucleus"; and "autophagosome membrane", respectively, are not present in the top 60 descriptors and, with a cumulative gain of 0.003 , are not contributing to the model. This, most likely, has to do with lack of association between these terms and the 103 ATG-positive genes. LINCS-associated terms do not provide a significant contribution to the model. This is not surprising, since the LINCS descriptors used here are derived from measuring cancer cell genomic response to drug perturbation, which is perhaps less relevant in the context of autophagy. Among the top 40 descriptors are 11 PPI nodes: ATG12, MAPK1, ATG4B, BECN1, SQSTM1, PIK3CD, MARK2, MAPK15, AKT2, GABARAPL2, and TSC1, respectively, with the top 4 (ATG12, MAPK1, ATG4B, and BECN1) accounting for $40 \%$ of the total gain. Except for MARK2 and MAPK15, these form a complex network of protein interactions (https://bit.ly/2JHygyq). The vast majority of the STRING [30] analysis enrichment terms point to autophagy (including macroautophagy, autophagy of mitochondrion, autophagosome assembly); phosphotransferase and kinase activity; as well as five publications related to autophagy [51-55]. 


\section{Insert Figure 2}

Figure 2. Top 40 variables, selected by the ATG MPxgb model, in decreasing order of importance. PPI: protein-protein interaction nodes; IPR: InterPro domain; GO: Gene Ontology term; HPA: Human Protein Atlas. See text for additional details.

Of the two outlier PPI nodes, MARK2 and MAPK15, MAPK15 is part of the ULK complex [56] and stimulates autophagy by interacting with ATG8 family proteins [57]. The involvement of MARK2 in autophagy is less clear [58]. When combined with the 103 ATG-model input genes, a complex regulatory network enriched in the same autophagy terms is observed (https://bit.ly/2Z2SF60), with 3 additional KEGG pathways: "hsa04211: Longevity regulating pathway", "hsa04150: mTOR signaling pathway" and "hsa04068: FoxO signaling pathway", respectively. The top five publications are also related to autophagy [52,59-62]. Based on the STRING analysis of MPxgb selected PPI nodes, we conclude that these descriptors are relevant for autophagy. The MPxgb variable selection of IPR domains, as well as GO terms, is also related to genes that play a relevant role in autophagy (see also Supplementary Material). We conclude that the ATG MPxgb model discussed herein is significantly enriched in autophagy related terms and PPI nodes, and bears relevance for guiding future autophagy research with respect to potentially novel gene annotation for ATG involvement.

\section{Model Output: Quantifying Knowledge}

We examined the top 251 predicted genes, as ranked by the predicted probability of association with ATG. Among these, 9 were present in the initial set of 288 ATGdb genes; another 34 genes are annotated in the ATGdb expanded set (see Supplementary Material). A total of 43 genes predicted by the ATG MPxgb model were retrieved from ATGdb. Another set of 15 genes could be found by performing additional queries, such as "Autophagy Pathway" in PathCards [63]. Thus, 58 of the top 251 genes, or $23.1 \%$, appear to have confirmed (or strongly suspected) association with autophagy. However, 193 of these top 251 genes do not appear to be associated with autophagy, despite efforts to consult a variety of literature-based resources and online databases (which include ATGdb, PathCards, KEGG, GO, UniProt and TCRD).

Given that most of the top 40 ATG MPxgb variables and $23 \%$ of the top 251 genes (including the gene ranked 246, ARFGEF1 - present in ATGdb), we posit that this machine learning model bears relevance in the study of autophagy, and suggest that some of these 193 genes may represent autophagy "dark genes". We also examined the "Target development level" (TDL), a knowledge-based classification for human proteins [22] that can be used to explore the dark genome [64]. In brief, Tclin are proteins via which approved drugs act (i.e., mode-of-action drug targets); Tchem are proteins known to bind small molecules with high potency; Tbio are proteins with well-studied biology, having a fractional publication count above 5 [65] or well-annotated OMIM (disease) phenotypes; and Tdark are understudied proteins that do not meet criteria for the above 3 categories, respectively. The TDL count for the training set (103 ATG-associated genes), the test set (top 251 predicted genes) and the "ATG dark genes?" subset (193 
genes) is summarized in Table 2. As estimated by this knowledge classification system, it can be concluded that the majority of the predicted genes, specifically for the ATG dark genes, are either Tbio or Tdark. Indeed, nearly $30.6 \%$ of the "ATG dark genes" are classified as Tdark. which suggests that a significant portion of these genes are understudied.

Table 2. Target development level distribution for the ATG MPxgb model sets

$\begin{array}{lrrrr}\text { Set } & \text { Tclin } & \text { Tchem } & \text { Tbio } & \text { Tdark } \\ \text { Training } & 10 & 32 & 60 & 1 \\ \text { Test } & 0 & 52 & 137 & 62 \\ \text { ATG dark gene? } & 0 & 37 & 97 & 59\end{array}$

\section{Model Output: Newly Predicted Genes}

For the sake of brevity, we focus the remainder of this discussion on the top 40 predicted genes, which are outlined in Table 3. Of these, 15 genes are already in ATGdb (GABARAPL3, ULK3, EIF2AK2, RRAGD, RAB1A, VPS39, CSNK1G2, SNX1, CSNK2A2, PRKAB1, RAB9A, PRKCI, MAP3K14, EXOC4, CALCOCO2). RMDN1, interacts with Beclin 1, a known regulator of autophagy [66]; another gene, SLK, escorts VPS4B which itself is involved in autophagy [67]; OXSR1 is upstream of AMPK, a known autophagy regulator [68]; whereas MTMR3 and BNIP3L are ATG-annotated in PathCards.

Twenty of the top 40 genes may represent, however, novel assertions with respect to autophagy: MAP1LC3B2, TAOK3, NEK6, CLK1, STK35, NLK, VPS4A, RNF41, CLK3, VTI1A, RAB24, UBALD1, S100A6, PRKD2, NRBP1, CSNK1A1, RBM18, IKBKE, GRK5, and TMEM167A, respectively. With six exceptions (TAOK3, CLK1, CLK3, UBALD1, RBM18, and TMEM167A), the remaining 14 genes form a complex network when combined with the $11 \mathrm{PPI}$ nodes discussed in the Variable Importance section (https://bit.ly/2Yc5EoZ). As previously mentioned, the STRING analysis enrichment terms point to autophagy (including autophagy, macroautophagy, and autophagosome assembly); protein serine/threonine kinase activity activity; KEGG Pathway terms "hsa04140: Autophagy - animal", "hsa05167: Kaposi's sarcoma-associated herpesvirus infection", "hsa05160: Hepatitis C" and "hsa04152: AMPK signaling pathway" as well as multiple publications related to autophagy $[52,53,55,60,69-72]$.

Since many of the potentially novel 20 genes out of the top 40 are kinases, we used $\mathrm{X} 2 \mathrm{~K}$ Web (eXpression2Kinases) [73] to perform a Transcription Factor Enrichment Analysis (TFEA) [74]. This type of analysis predicts which transcription factors (TFs) are most likely to regulate the expression of these 20 genes. The ranked list of predicted TFs is visually summarized in Figure 3. The top ranked 13 transcription factors, selected to ensure that each of the top 20 predicted autophagy genes is represented at least once, are as follows: RUNX1, TCF7L2, ELF1, TAF1, GATA1, FOXP2, FOXA1, SPI1, CHD1, SRF, FOXA2, ZNF384, and BRCA1, respectively.

When combining the 11 PPI node genes selected by the ATG MPxgb model with the 13 genes TFEAselected genes, the top potentially novel ATG genes form a complex STRING-based network 
(https://bit.ly/2YeOffr) that leaves six genes out (NRBP1, CLK1,CLK3, UBALD1, RBM18, and TMEM167A). In addition to GO terms related to autophagy, the STRING analysis enrichment terms include "transcription factor binding" and "membrane-bounded organelle"; the UniProt keyword "Phosphoprotein"; the KEGG Pathway terms "hsa03022: Basal transcription factors", "hsa04137:

Mitophagy - animal" and "hsa05168: Herpes simplex infection", as well as several publications related to autophagy $[51,52,55,69,75-77]$. While the TFEA step does appear to include STRING enrichment terms associated to TFs, it also provides additional data elements supporting the role of some proteins left out of the STRING-based PPI network: UBALD1 might be regulated by 10 of the 13 TFs, TAOK3 and CLK1 by 5 , and TMEM167A by 4 TFs, whereas NRBP1 and RBM18 are regulated by 2 . Both this and the prior STRING network highlight the relationship between some ATG-related genes and viral $(H$. simplex , Hepatitis $C$ ) infection. This is not surprising, since autophagy is an antiviral defense mechanism.

\section{Insert Figure 3}

Figure 3. Visual summary of the transcription factor enrichment analysis for the top 20 predicted genes autophagy dark genes. Thirteen transcription factors are shown in reverse order of P-value ranking. See text for additional details. 
Table 3. List of top 40 genes from the ATG MPxgb model. HUGO Gene Nomenclature Committee. Pred.prob - predicted probability, as derived from the model.

\begin{tabular}{|c|c|c|c|c|c|}
\hline $\begin{array}{l}\text { UniProt } \\
\text { Accession }\end{array}$ & $\begin{array}{l}\text { HGCN } \\
\text { symbol }\end{array}$ & Protein name & pred.prob & TDL & Autophagy Information \\
\hline Q9BY60 & GABARAPL3 & $\begin{array}{l}\text { Gamma-aminobutyric acid receptor-associated } \\
\text { protein-like } 3\end{array}$ & 0.967 & Tdark & Present in ATGdb (288 set) \\
\hline Q96DB5 & RMDN1 & Regulator of microtubule dynamics protein 1 & 0.917 & Tdark & Beclin interacting / STRING-DB \\
\hline P19525 & EIF2AK2 & $\begin{array}{l}\text { Interferon-induced, double-stranded RNA- } \\
\text { activated protein kinase }\end{array}$ & 0.910 & Tchem & Present in ATGdb (expanded set) \\
\hline Q9H2G2 & SLK & STE20-like serine/threonine-protein kinase & 0.770 & Tchem & escort VPS4B \\
\hline Q13615 & MTMR3 & Myotubularin-related protein 3 & 0.749 & Tbio & $\begin{array}{l}\text { Autophagy Pathway PathCards|Autophagy } \\
\text { animal PathCards }\end{array}$ \\
\hline P62820 & RAB1A & Ras-related protein Rab-1A & 0.731 & Tbio & Present in ATGdb (expanded set) \\
\hline A6NCE7 & MAP1LC3B2 & $\begin{array}{l}\text { Microtubule-associated proteins } 1 \mathrm{~A} / 1 \mathrm{~B} \text { light } \\
\text { chain } 3 \text { beta } 2\end{array}$ & 0.713 & Tdark & ATG dark gene? \\
\hline O60238 & BNIP3L & $\begin{array}{l}\text { BCL2/adenovirus E1B } 19 \mathrm{kDa} \text { protein- } \\
\text { interacting protein 3-like }\end{array}$ & 0.683 & Tbio & Autophagy Pathway PathCards \\
\hline Q8TDR2 & STK35 & Serine/threonine-protein kinase 35 & 0.676 & Tchem & ATG dark gene? \\
\hline P78368 & CSNK1G2 & Casein kinase I isoform gamma-2 & 0.675 & Tchem & Present in ATGdb (expanded set) \\
\hline Q9UBE8 & NLK & Serine/threonine-protein kinase NLK & 0.652 & Tchem & ATG dark gene? \\
\hline Q9UN37 & VPS4A & Vacuolar protein sorting-associated protein 4A & 0.651 & Tbio & ATG dark gene? \\
\hline Q9H4P4 & RNF41 & E3 ubiquitin-protein ligase NRDP1 & 0.630 & Tbio & ATG dark gene? \\
\hline Q13596 & SNX1 & Sorting nexin-1 & 0.619 & Tbio & Present in ATGdb (288 set) \\
\hline P49761 & CLK3 & Dual specificity protein kinase CLK3 & 0.585 & Tchem & ATG dark gene? \\
\hline Q96AJ9 & VTI1A & Vesicle transport through interaction with t- & 0.585 & Tbio & ATG dark gene? \\
\hline
\end{tabular}




\begin{tabular}{|c|c|c|c|c|c|}
\hline P19784 & CSNK2A2 & Casein kinase II subunit alpha' & 0.577 & Tchem & Present in ATGdb (expanded set) \\
\hline Q9Y478 & PRKAB1 & 5'-AMP-activated protein kinase subunit beta-1 & 0.576 & Tchem & Present in ATGdb (expanded set) \\
\hline P51151 & RAB9A & Ras-related protein Rab-9A & 0.518 & Tbio & Present in ATGdb (expanded set) \\
\hline Q969Q5 & RAB24 & Ras-related protein Rab-24 & 0.517 & Tbio & ATG dark gene? \\
\hline P41743 & PRKCl & Protein kinase $C$ iota type & 0.512 & Tchem & Present in ATGdb (expanded set) \\
\hline Q8TB05 & UBALD1 & UBA-like domain-containing protein 1 & 0.496 & Tdark & ATG dark gene? \\
\hline P06703 & S100A6 & Protein S100-A6 & 0.468 & Tbio & ATG dark gene? \\
\hline Q9BZL6 & PRKD2 & Serine/threonine-protein kinase D2 & 0.462 & Tchem & ATG dark gene? \\
\hline Q99558 & MAP3K14 & $\begin{array}{l}\text { Mitogen-activated protein kinase kinase kinase } \\
14\end{array}$ & 0.453 & Tchem & Present in ATGdb (expanded set) \\
\hline Q9UHY1 & NRBP1 & Nuclear receptor-binding protein & 0.437 & Tbio & ATG dark gene? \\
\hline P48729 & CSNK1A1 & Casein kinase I isoform alpha & 0.436 & Tchem & ATG dark gene? \\
\hline Q96H35 & RBM18 & Probable RNA-binding protein 18 & 0.430 & Tdark & ATG dark gene? \\
\hline Q96A65 & EXOC4 & Exocyst complex component 4 & 0.415 & Tbio & Present in ATGdb (expanded set) \\
\hline Q14164 & IKBKE & $\begin{array}{l}\text { Inhibitor of nuclear factor kappa-B kinase } \\
\text { subunit epsilon }\end{array}$ & 0.402 & Tchem & ATG dark gene? \\
\hline P34947 & GRK5 & G protein-coupled receptor kinase 5 & 0.395 & Tchem & ATG dark gene? \\
\hline Q8TBQ9 & TMEM167A & Protein kish-A & 0.394 & Tdark & ATG dark gene? \\
\hline Q13137 & CALCOCO2 & $\begin{array}{l}\text { Calcium-binding and coiled-coil domain- } \\
\text { containing protein } 2\end{array}$ & 0.394 & Tbio & Present in ATGdb (expanded set) \\
\hline
\end{tabular}




\section{Conclusions}

In this paper we introduced an ATG-dedicated machine learning model, which we intended to provide as guidance for extending current autophagy knowledge, in order to explore gene that previously have not been evaluated for their role in autophagy. In developing this ATG-specific MPxgb model, we started with 288 genes associated with autophagy from the Autophagy database, ATGdb [49], upon which we set additional filters (association with GO, KEGG and UniProt terms), to increase confidence in the remaining 103 positive label genes. As negative labels, we used $N=3,468$ genes associated with monogenic diseases in OMIM, which were not present in ATGdb, as genes lacking the ATG association. This assumption, which is necessary for many machine learning methods, specifically for binary classifiers, may represent a significant flaw since we lack absolute certainty that negative examples are not genes playing a role in autophagy. However, just as any other ML models, the ATG-specific MPxgb model requires extensive validation, perhaps via several iterations. Reflecting current levels of evidence, we used XGBoost, a binary classifier combined with the Metapath (Figure 2) approach, which was deployed upon 17 sources of data representing seven distinct categories (Table 1). The ATG MPxgb model implicates up to 193 previously untested genes that may have a functional role in autophagy. This was, in fact, our primary motivation: to provide the scientific community with a ML-ready list of autophagy genes, as summarized by the training set, combined with a truly blind prediction set $(N=193)$. By disclosing this list of putative genes, we aim to encourage further experimental testing. All gene sets mentioned throughout this paper are available in the Supplementary Material. Future validation steps may include integration of chemical-protein annotation resources, using Pharos [48] and Chem-Prot [78], the use of a semantic model to evaluate druggability via the drug target ontology [79].

\section{Acknowledgments}

This work was supported by NIH grants U24 CA224370 (TIO, DB, JJY), U24 TR002278 (TIO) and P20 GM121176 (TIO, VD). Dr. Oleg Ursu (Merck Research Laboratories) implemented the initial version of the MPxgb algorithm.

\section{References}

1. Mizushima N, Yoshimori T, Ohsumi Y. The role of Atg proteins in autophagosome formation. Annu Rev Cell Dev Biol. 2011;27: 107-132.

2. Mizushima N, Levine B, Cuervo AM, Klionsky DJ. Autophagy fights disease through cellular selfdigestion. Nature. 2008;451: 1069-1075.

3. Levine B, Kroemer G. Biological Functions of Autophagy Genes: A Disease Perspective. Cell. 
2019;176: 11-42.

4. Hosokawa N, Sasaki T, lemura S-I, Natsume T, Hara T, Mizushima N. Atg101, a novel mammalian autophagy protein interacting with Atg13. Autophagy. 2009;5: 973-979.

5. Hara T, Takamura A, Kishi C, lemura S-I, Natsume T, Guan J-L, et al. FIP200, a ULK-interacting protein, is required for autophagosome formation in mammalian cells. J Cell Biol. 2008;181: 497510.

6. Ropolo A, Grasso D, Pardo R, Sacchetti ML, Archange C, Lo Re A, et al. The pancreatitis-induced vacuole membrane protein 1 triggers autophagy in mammalian cells. J Biol Chem. 2007;282: 3712437133.

7. Wang Z, Miao G, Xue X, Guo X, Yuan C, Wang Z, et al. The Vici Syndrome Protein EPG5 Is a Rab7 Effector that Determines the Fusion Specificity of Autophagosomes with Late Endosomes/Lysosomes. Mol Cell. 2016;63: 781-795.

8. Itakura E, Kishi-Itakura C, Mizushima N. The hairpin-type tail-anchored SNARE syntaxin 17 targets to autophagosomes for fusion with endosomes/lysosomes. Cell. 2012;151: 1256-1269.

9. Hamasaki M, Furuta N, Matsuda A, Nezu A, Yamamoto A, Fujita N, et al. Autophagosomes form at ER-mitochondria contact sites. Nature. 2013;495: 389-393.

10. Moretti F, Bergman P, Dodgson S, Marcellin D, Claerr I, Goodwin JM, et al. TMEM41B is a novel regulator of autophagy and lipid mobilization. EMBO Rep. 2018;19. doi:10.15252/embr.201845889

11. Morita K, Hama Y, Izume T, Tamura N, Ueno T, Yamashita Y, et al. Genome-wide CRISPR screen identifies TMEM41B as a gene required for autophagosome formation. J Cell Biol. 2018;217: 38173828.

12. Shoemaker CJ, Huang TQ, Weir NR, Polyakov NJ, Schultz SW, Denic V. CRISPR screening using an expanded toolkit of autophagy reporters identifies TMEM41B as a novel autophagy factor. PLoS Biol. 2019;17: e2007044.

13. Terman A, Gustafsson B, Brunk UT. Autophagy, organelles and ageing. J Pathol. 2007;211: 134143.

14. Hars ES, Qi H, Ryazanov AG, Jin S, Cai L, Hu C, et al. Autophagy regulates ageing in C. elegans. Autophagy. 2007;3: 93-95.

15. Vellai T. Autophagy genes and ageing. Cell Death Differ. 2009;16: 94-102.

16. Fabris F, Magalhães JP de, Freitas AA. A review of supervised machine learning applied to ageing research. Biogerontology. 2017;18: 171-188.

17. Barardo DG, Newby D, Thornton D, Ghafourian T, de Magalhães JP, Freitas AA. Machine learning for predicting lifespan-extending chemical compounds. Aging . 2017;9: 1721-1737.

18. Radivojac P, Peng K, Clark WT, Peters BJ, Mohan A, Boyle SM, et al. An integrated approach to inferring gene-disease associations in humans. Proteins. 2008;72: 1030-1037.

19. Mordelet F, Vert J-P. ProDiGe: Prioritization Of Disease Genes with multitask machine learning from positive and unlabeled examples. BMC Bioinformatics. 2011;12: 389.

20. Yang P, Li X, Chua H-N, Kwoh C-K, Ng S-K. Ensemble positive unlabeled learning for disease gene identification. PLoS One. 2014;9: e97079.

21. Lan W, Wang J, Li M, Peng W, Wu F. Computational approaches for prioritizing candidate disease 
genes based on PPI networks. Tsinghua Sci Technol. 2015;20: 500-512.

22. Oprea TI, Bologa CG, Brunak S, Campbell A, Gan GN, Gaulton A, et al. Unexplored therapeutic opportunities in the human genome. Nat Rev Drug Discov. 2018;17: 377.

23. Huerta-Cepas J, Szklarczyk D, Forslund K, Cook H, Heller D, Walter MC, et al. eggNOG 4.5: a hierarchical orthology framework with improved functional annotations for eukaryotic, prokaryotic and viral sequences. Nucleic Acids Res. 2016;44: D286-93.

24. Sonnhammer ELL, Östlund G. InParanoid 8: orthology analysis between 273 proteomes, mostly eukaryotic. Nucleic Acids Res. 2015;43: D234-9.

25. Barretina J, Caponigro G, Stransky N, Venkatesan K, Margolin AA, Kim S, et al. The Cancer Cell Line Encyclopedia enables predictive modelling of anticancer drug sensitivity. Nature. 2012;483: 603-607.

26. Wheeler HE, Shah KP, Brenner J, Garcia T, Aquino-Michaels K, GTEx Consortium, et al. Survey of the Heritability and Sparse Architecture of Gene Expression Traits across Human Tissues. PLoS Genet. 2016;12: e1006423.

27. Uhlén M, Fagerberg L, Hallström BM, Lindskog C, Oksvold P, Mardinoglu A, et al. Proteomics. Tissue-based map of the human proteome. Science. 2015;347: 1260419.

28. Fabregat A, Sidiropoulos K, Garapati P, Gillespie M, Hausmann K, Haw R, et al. The Reactome pathway Knowledgebase. Nucleic Acids Res. 2016;44: D481-7.

29. Kanehisa M, Furumichi M, Tanabe M, Sato Y, Morishima K. KEGG: new perspectives on genomes, pathways, diseases and drugs. Nucleic Acids Res. 2017;45: D353-D361.

30. Szklarczyk D, Morris JH, Cook H, Kuhn M, Wyder S, Simonovic M, et al. The STRING database in 2017: quality-controlled protein-protein association networks, made broadly accessible. Nucleic Acids Res. 2017;45: D362-D368.

31. The Gene Ontology Consortium. Expansion of the Gene Ontology knowledgebase and resources. Nucleic Acids Res. 2017;45: D331-D338.

32. Finn RD, Attwood TK, Babbitt PC, Bateman A, Bork P, Bridge AJ, et al. InterPro in 2017-beyond protein family and domain annotations. Nucleic Acids Res. 2017;45: D190-D199.

33. Landrum MJ, Lee JM, Benson M, Brown G, Chao C, Chitipiralla S, et al. ClinVar: public archive of interpretations of clinically relevant variants. Nucleic Acids Res. 2016;44: D862-8.

34. Welter D, MacArthur J, Morales J, Burdett T, Hall P, Junkins H, et al. The NHGRI GWAS Catalog, a curated resource of SNP-trait associations. Nucleic Acids Res. 2014;42: D1001-6.

35. OMIM - Online Mendelian Inheritance in Man [Internet]. [cited 22 Mar 2017]. Available: https://omim.org/

36. The UniProt Consortium. UniProt: the universal protein knowledgebase. Nucleic Acids Res. 2017;45: D158-D169.

37. Pletscher-Frankild S, Pallejà A, Tsafou K, Binder JX, Jensen LJ. DISEASES: Text mining and data integration of disease-gene associations [Internet]. 2014. doi:10.1101/008425

38. Homology - Site Guide - NCBI [Internet]. [cited 18 Jul 2019]. Available:

https://www.ncbi.nlm.nih.gov/guide/homology/

39. Bradley A, Anastassiadis K, Ayadi A, Battey JF, Bell C, Birling M-C, et al. The mammalian gene 
function resource: the International Knockout Mouse Consortium. Mamm Genome. 2012;23: 580586.

40. Shimoyama M, De Pons J, Hayman GT, Laulederkind SJF, Liu W, Nigam R, et al. The Rat Genome Database 2015: genomic, phenotypic and environmental variations and disease. Nucleic Acids Res. 2015;43: D743-50.

41. Niepel M, Hafner M, Mills CE, Subramanian K, Williams EH, Chung M, et al. A Multi-center Study on the Reproducibility of Drug-Response Assays in Mammalian Cell Lines. Cell Syst. 2019; doi:10.1016/j.cels.2019.06.005

42. Sun Y, Barber R, Gupta M, Aggarwal CC, Han J. Co-author Relationship Prediction in Heterogeneous Bibliographic Networks. 2011 International Conference on Advances in Social Networks Analysis and Mining. 2011. doi:10.1109/asonam.2011.112

43. Liang W, He X, Tang D, Zhang X. S-Rank: A Supervised Ranking Framework for Relationship Prediction in Heterogeneous Information Networks. Lecture Notes in Computer Science. 2016. pp. 305-319.

44. Himmelstein DS, Baranzini SE. Heterogeneous Network Edge Prediction: A Data Integration Approach to Prioritize Disease-Associated Genes. PLoS Comput Biol. 2015;11: e1004259.

45. Fu G, Ding Y, Seal A, Chen B, Sun Y, Bolton E. Predicting drug target interactions using meta-pathbased semantic network analysis. BMC Bioinformatics. 2016;17: 160.

46. Sun Y, Han J, Aggarwal CC, Chawla NV. When will it happen?: relationship prediction in heterogeneous information networks. Proceedings of the fifth ACM international conference on Web search and data mining. ACM; 2012. pp. 663-672.

47. Chen T, Guestrin C. XGBoost: A Scalable Tree Boosting System. Proceedings of the 22Nd ACM SIGKDD International Conference on Knowledge Discovery and Data Mining. New York, NY, USA: ACM; 2016. pp. 785-794.

48. Nguyen D-T, Mathias S, Bologa C, Brunak S, Fernandez N, Gaulton A, et al. Pharos: Collating protein information to shed light on the druggable genome. Nucleic Acids Res. 2017;45: D995D1002.

49. Homma K, Suzuki K, Sugawara H. The Autophagy Database: an all-inclusive information resource on autophagy that provides nourishment for research. Nucleic Acids Res. 2011;39: D986-90.

50. Bennett J, Lanning S, Netflix N. The Netflix Prize. In KDD Cup and Workshop in conjunction with KDD. 2009. Available: http://dx.doi.org/

51. Rosenfeldt MT, Ryan KM. The role of autophagy in tumour development and cancer therapy. Expert Rev Mol Med. 2009;11: e36.

52. Kroemer G, Mariño G, Levine B. Autophagy and the integrated stress response. Mol Cell. 2010;40: 280-293.

53. Ding W-X, Manley S, Ni H-M. The emerging role of autophagy in alcoholic liver disease. Exp Biol Med . 2011;236: 546-556.

54. Dumit VI, Dengjel J. Autophagosomal protein dynamics and influenza virus infection. Front Immunol. 2012;3: 43.

55. Hensley K, Harris-White ME. Redox regulation of autophagy in healthy brain and neurodegeneration. Neurobiol Dis. 2015;84: 50-59. 
56. Colecchia D, Dapporto F, Tronnolone S, Salvini L, Chiariello M. MAPK15 is part of the ULK complex and controls its activity to regulate early phases of the autophagic process. J Biol Chem. 2018;293: 15962-15976.

57. Colecchia D, Strambi A, Sanzone S, lavarone C, Rossi M, Dall'Armi C, et al. MAPK15/ERK8 stimulates autophagy by interacting with LC3 and GABARAP proteins. Autophagy. 2012;8: 17241740.

58. Rigbolt KT, Zarei M, Sprenger A, Becker AC, Diedrich B, Huang X, et al. Characterization of early autophagy signaling by quantitative phosphoproteomics. Autophagy. 2014;10: 356-371.

59. Klionsky DJ, Codogno P, Cuervo AM, Deretic V, Elazar Z, Fueyo-Margareto J, et al. A comprehensive glossary of autophagy-related molecules and processes. Autophagy. 2010;6: 438448.

60. Liu B, Cheng Y, Liu Q, Bao J-K, Yang J-M. Autophagic pathways as new targets for cancer drug development. Acta Pharmacol Sin. 2010;31: 1154-1164.

61. Yang Z, Klionsky DJ. Mammalian autophagy: core molecular machinery and signaling regulation. Curr Opin Cell Biol. 2010;22: 124-131.

62. Reggiori F. Autophagy: New Questions from Recent Answers. ISRN Mol Biol. 2012;2012: 738718.

63. Belinky F, Nativ N, Stelzer G, Zimmerman S, Iny Stein T, Safran M, et al. PathCards: multi-source consolidation of human biological pathways. Database . 2015;2015. doi:10.1093/database/bav006

64. Oprea TI. Exploring the dark genome: implications for precision medicine. Mamm Genome. 2019; doi:10.1007/s00335-019-09809-0

65. Pafilis E, Frankild SP, Fanini L, Faulwetter S, Pavloudi C, Vasileiadou A, et al. The SPECIES and ORGANISMS Resources for Fast and Accurate Identification of Taxonomic Names in Text. PLoS One. 2013;8: e65390.

66. Vergne I, Deretic $\mathrm{V}$. The role of PI3P phosphatases in the regulation of autophagy. FEBS Lett. 2010;584: 1313-1318.

67. Shrivastava S, Devhare P, Sujijantarat N, Steele R, Kwon Y-C, Ray R, et al. Knockdown of Autophagy Inhibits Infectious Hepatitis C Virus Release by the Exosomal Pathway. J Virol. 2016;90: 1387-1396.

68. Kim J, Kundu M, Viollet B, Guan K-L. AMPK and mTOR regulate autophagy through direct phosphorylation of Ulk1. Nat Cell Biol. 2011;13: 132-141.

69. Wani WY, Boyer-Guittaut M, Dodson M, Chatham J, Darley-Usmar V, Zhang J. Regulation of autophagy by protein post-translational modification. Lab Invest. 2015;95: 14-25.

70. Su Z, Yang Z, Xu Y, Chen Y, Yu Q. MicroRNAs in apoptosis, autophagy and necroptosis. Oncotarget. 2015;6: 8474-8490.

71. Liu G, Pei F, Yang F, Li L, Amin AD, Liu S, et al. Role of Autophagy and Apoptosis in Non-Small-Cell Lung Cancer. Int J Mol Sci. 2017;18. doi:10.3390/ijms18020367

72. Budini M, Buratti E, Morselli E, Criollo A. Autophagy and Its Impact on Neurodegenerative Diseases: New Roles for TDP-43 and C9orf72. Front Mol Neurosci. 2017;10: 170.

73. Clarke DJB, Kuleshov MV, Schilder BM, Torre D, Duffy ME, Keenan AB, et al. eXpression2Kinases $(\mathrm{X} 2 \mathrm{~K})$ Web: linking expression signatures to upstream cell signaling networks. Nucleic Acids Res. 2018;46: W171-W179. 
74. Lachmann A, Xu H, Krishnan J, Berger SI, Mazloom AR, Ma'ayan A. ChEA: transcription factor regulation inferred from integrating genome-wide ChIP-X experiments. Bioinformatics. 2010;26: 2438-2444.

75. Reggiori F, Komatsu M, Finley K, Simonsen A. Autophagy: more than a nonselective pathway. Int J Cell Biol. 2012;2012: 219625.

76. Ávalos Y, Canales J, Bravo-Sagua R, Criollo A, Lavandero S, Quest AFG. Tumor suppression and promotion by autophagy. Biomed Res Int. 2014;2014: 603980.

77. Gumeni S, Evangelakou Z, Gorgoulis VG, Trougakos IP. Proteome Stability as a Key Factor of Genome Integrity. Int J Mol Sci. 2017;18. doi:10.3390/ijms18102036

78. Taboureau O, Nielsen SK, Audouze K, Weinhold N, Edsgärd D, Roque FS, et al. ChemProt: a disease chemical biology database. Nucleic Acids Res. 2011;39: D367-72.

79. Lin Y, Mehta S, Küçük-McGinty H, Turner JP, Vidovic D, Forlin M, et al. Drug target ontology to classify and integrate drug discovery data. J Biomed Semantics. 2017;8: 50. 


\section{Variable Importance}
0
0.05
0.1
0.15
0.2
0.25

PPI:ATG12

IPR: Immunoglobulin-like fold

IPR: Prote in kinase domain

PPI:MAPK1

bioRxiv preprint doi: https://doi.org/10.1101/715037; this version posted July 26, 2019. The copytight holderfor-this preprint (which was not certified by peer review) is the author/funder, who has granted bioRxiv a license to display the preprint in-perpetuity. It is made available under aCC-BY 4.0 International license. 1. Al G4B
GO: Macroautophagy
GO: autophagosome
PPI:BECN1

IPR: Protein kinase, ATP binding site

GO: autophagosome assembly

GO: cytoplasmic vesicle

GO: protein binding

PPI:SQSTM1

PPI:PIK3CD

CCLE: LUNG (NCIH1930)

LINCS: lomifylline:A549 signature GO: cellular response to amino acid... LINCS: im iquimod:HCT116 signature PPI:MARK2

KEGG: Metabolic pathways

LINCS: ixazomib:NOMO1 signature

KEGG: Cell cycle

GO: regulation of mac roautophagy

PPI:MAPK15

GO: regulation of autophagy

LINCS: tamibarotene:VCAP signature

PPI:AKT2

PPI:GABARAPL2

HPA: cytosol

REACTOME: Signal ing by ROBO receptors LINCS: mebendazole:VCAP signature PPI:TSC1

LINCS: venl afaxine:HEPG2 signature LINCS: tol naftate:HA1E signature LINCS: dichlorphenamide:VCAP signature HPA: plasma membrane IPR: Protein kinase-like domain superfamily UniProt: pre-autophagosomal structure LINCS: menadione:SNUC4 signature 
$\begin{array}{lllllllllll}\text { Gene } & \text { RUNX1 TCF7L2 ELF1 TAF1 GATA1 FOXP2 FOXA1 SPI } & \text { CHD1 } & \text { SRF FOXA2 ZNF384 BRCA1 }\end{array}$ MAP1LC3B2

TAOK3

NEK6

CLK1

STK35

NLK

VPS4A

RNF41

CLK3

VTI1A

RAB24

UBALD1

S100A6

PRKD2

NRBP1

CSNK1A1

RBM18

IKBKE

GRK5

TMEM167A
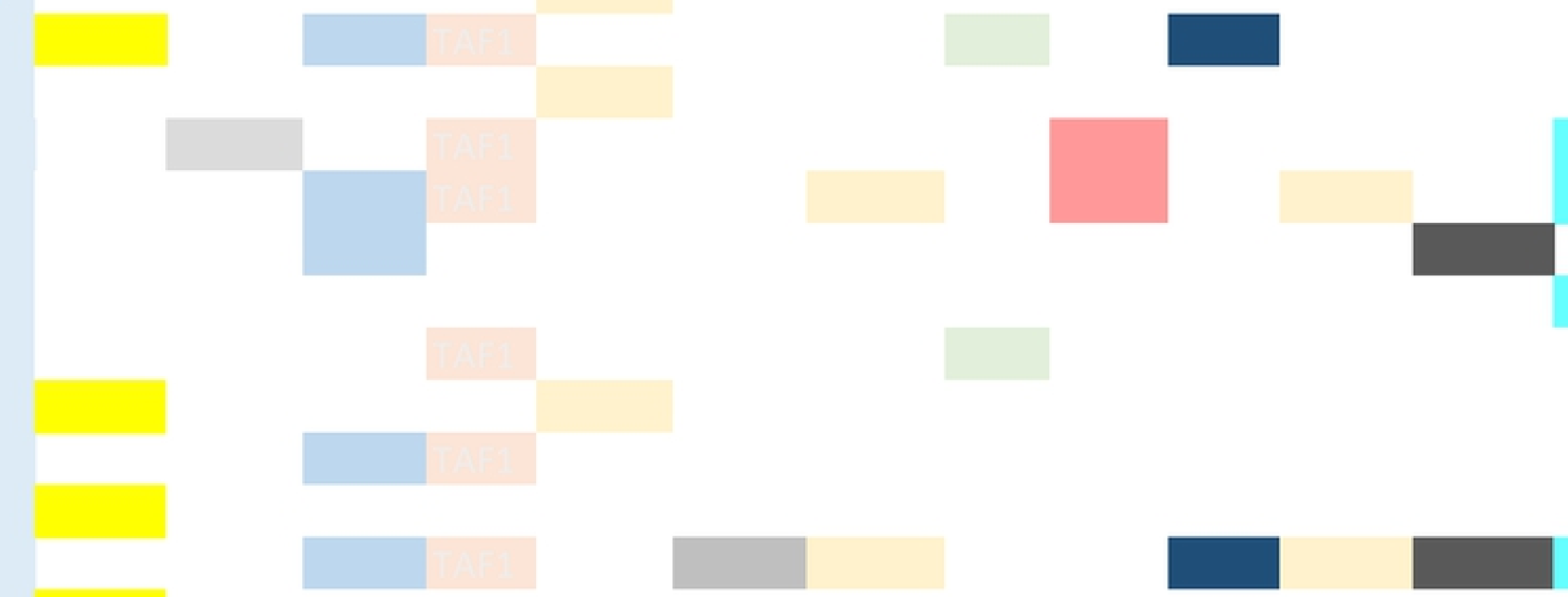

P-value

$\begin{array}{llll}0.005 & 0.006 & 0.009 & 0.016\end{array}$

0.018

0.022

$\begin{array}{llll}0.029 & 0.043 & 0.050 & 0.056\end{array}$

0.062

0.065

0.101

Figure 3 